\title{
Assessment of excision of basal cell carcinoma: did GPs really 'underperform'?
}

\author{
Author: Syukri Rahim
}

\section{Aims}

Much of the work on basal cell carcinoma (BCC) in the literature mainly focused on the assessment of BCC excision completeness and comparing the performance of GPs, plastic surgeons and dermatologists. While most studies have consistently reported that GPs tended to do less well than the other specialists, little or no work was done to explore this even further.

The study aimed: 1) to assess the performance of GPs, dermatologists and plastic surgeons in excision of basal cell carcinoma. 2) To identify factors or predictors of excision completeness. 3) To identify certain patterns of excision made by each specialty, including excision cut margin, size of lesion, confidence in diagnosing skin lesion, etc.

\section{Methods}

Retrospective analysis of BCC excised in the Grampian region received by the pathology laboratory between 1 January 2011 and 1 December 2012.

Data obtained included age, gender, site of lesion, histological clearance by depth and radial margin, surgical cut margin, the specialty performing the excision and accuracy of diagnosing BCC based on written descriptions on request forms (abstract diagnosis)

Excision was considered complete and adequate if both radial and deep margins were $>1 \mathrm{~mm}$.

Statistical analyses were done using SPSS version 20. Logistic regression was used to determine the predictors of excision completeness.

\section{Results}

366 primary excisional BCC specimens were included in the current dataset. In the present dataset, GPs also underperformed with a $34.1 \%$ incomplete excision rate, compared with $7.8 \%$ in dermatologists and $26.0 \%$ in plastic surgeons. Based on the multivariate analysis, three independent variables significantly predicted excision completeness (cut margin, site of the lesion and specialty who performed the excision). Every $1 \mathrm{~mm}$ increase in the surgical cut margin increased the excision completeness by 1.31 times. Further investigation into this revealed that most GPs tended to perform with a smaller cut margin of $2 \mathrm{~mm}$ and were associated with uncertainty with the clinical diagnosis.

\section{Conclusions}

In the current dataset, while GPs also did not do 'as well as' dermatologists, this was mainly contributed by the fact that GPs tended to excise BCC with a significantly smaller cut margin. Comparable to past studies, GPs had the correct diagnosis in $68.3 \%$ of the cases, lower than dermatologists and plastic surgeons ( $95.6 \%$ and $82.8 \%$ respectively). Correctly diagnosing the lesion, however, did not appear to contribute significantly to excision completeness. 